\title{
Visibilidad e impacto de la producción científica de la Facultad de Ingeniería Industrial de la Cujae de Cuba (2003-2012)
}

\author{
María Sonia Fleitas Triana* \\ Claudia Hernández Oquendo** \\ Sandra Guerra Castillo***
}

Artículo recibido:

21 de octubre de 2013

Artículo aceptado:

29 de abril de 2016

\section{RESUMEN}

Los estudios bibliométricos aplicados a la producción científica de las universidades aportan información valiosa para la toma de decisiones en materia de planificación y evaluación de sus actividades de investigación. El objetivo de este artículo es representar y analizar el perfil investigador de la Facultad de Ingeniería Industrial del Instituto Superior Politécnico José Antonio Echeverría (Cujae) de Cuba, a partir de un estudio bibliométrico de la producción científica de

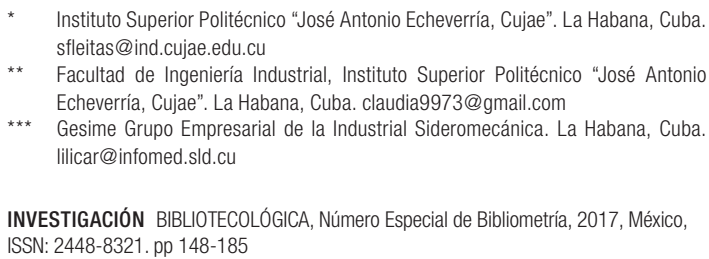




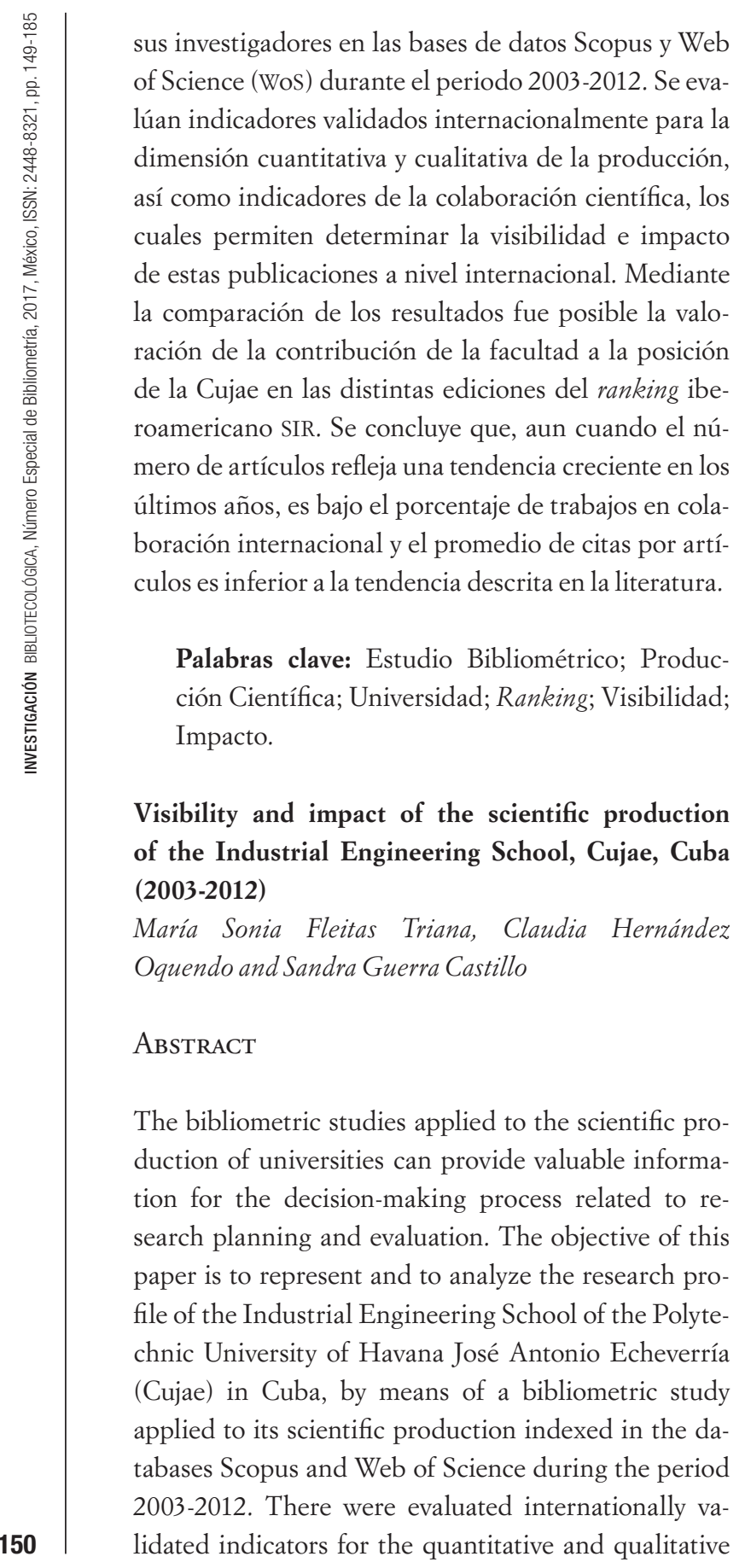


dimension of the production, as well as indicators for the scientific collaboration, which allowed the determination of the visibility and impact of these publications at international level. By means of the comparison of the results, it was possible the assessment of the contribution of the School to the position of Cujae in the several editions of the SIR Latin American ranking.

It was concluded that, even though there has been a positive tendency in the number of articles in the last years, the percentage of papers in international collaboration is low and the average of cites per paper is below the tendency described in the literature.

Key words: Bibliometric Study; Scientific Production; University; Ranking; Visibility; Impact.

\section{INTRODUCCIÓN}

La búsqueda de la visibilidad es un aspecto primordial para que la investigación logre un impacto adecuado en la comunidad científica y académica nacional e internacional, que es, sin lugar a duda, el objetivo que se persigue cuando se publican los resultados de la actividad científica de una institución o un país. Sin embargo, la brecha entre los países desarrollados y los subdesarrollados respecto del acceso, creación y utilización de los conocimientos científicos, han influido significativamente en la poca visibilidad internacional e impacto de la actividad científica de estos últimos.

En este escenario, la Política Nacional de Ciencia e Innovación Tecnológica, trazada por el Ministerio de Ciencia, Tecnología y Medio Ambiente (Citma) de Cuba, se manifiesta por la necesidad de asegurar el tratamiento adecuado de los resultados del trabajo científico y tecnológico dentro del sistema de publicaciones científicas nacionales, promoviendo, en la medida conveniente, la divulgación de trabajos nacionales en revistas de circulación internacional de reconocido prestigio.

El sector universitario, productor y diseminador principal del conocimiento en una sociedad, desempeña un papel protagónico en la actividad científica de cualquier nación. De acuerdo con los lineamientos de la política científica del país, el Ministerio de Educación Superior (MES) de Cuba tiene entre sus retos impulsar la producción científica de sus instituciones, siendo uno de los pilares para la consecución de este objetivo la publicación de esta producción en revistas nacionales e internacionales, específicamente las 
de mayor visibilidad internacional que forman parte de la llamada "corriente principal" de la ciencia.

Por ello, en 2010, la Dirección de Ciencia y Técnica del MES, con claros objetivos evaluativos y atendiendo a la existencia de nuevas herramientas cienciométricas para el estudio de la producción científica nacional, realizó una clasificación de las bases de datos en cuatro grupos e incluyó a las revistas comprendidas en las bases de datos de Thomson Reuters (antes Institute for Scientific Information, isi), disponibles en línea a través del portal Web of Science (WoS) y en Scopus, creada por la editorial Elsevier; dentro del grupo 1, considerado el más importante núcleo de fuentes de información en el que deben divulgar sus resultados los científicos y académicos cubanos.

La Facultad de Ingeniería Industrial es una de las siete con que cuenta el Instituto Superior Politécnico José Antonio Echeverría (en lo sucesivo Cujae), de Cuba, donde se imparte la carrera de Ingeniería Industrial, acreditada como de Excelencia desde 2004. Al estudiar las publicaciones científicas de sus investigadores en los últimos diez años, a partir de los indicadores que conforman el "Modelo de referencia de investigaciones y posgrado", ${ }^{4}$ se concluye que, aunque ha habido un progreso en el cumplimiento de los artículos planificados en el grupo 2 (que reúne las bases de datos especializadas de reconocimiento internacional), la producción científica en bases de datos de corriente principal (pertenecientes al grupo 1) ha sido insuficiente en el periodo mencionado.

Según los informes del listado o clasificación (ranking) iberoamericano SIR (scimago Institutions Ranking), desarrollado por los investigadores del grupo español scimago, a partir de la producción científica presente en la base de datos Scopus, la posición de la Cujae en cuanto a las publicaciones en revistas científicas ha variado del lugar 280 al 354, entre más de mil instituciones iberoamericanas de educación superior, en las ediciones publicadas anualmente desde 2010 hasta 2012. En la edición de 2013, esta posición mejoró en relación con el periodo anterior, para ubicarse en el lugar 306, al incrementarse la producción científica en 38 artículos.

A pesar de que el uso de las clasificaciones en el sector de la educación superior no genera consenso y conduce a muchos debates sobre los beneficios y costos de su uso, su empleo como herramienta para el análisis de la actividad investigadora de las instituciones de educación superior (IES) se ha

4 Instrumento de evaluación que contiene una serie de indicadores para medir los resultados de la actividad investigativa, que incluye, entre otros, el número de artículos publicados en la Web of Science (WoS) y en otras bases de datos de reconocimiento internacional. Éste se elabora por el Vicerrectorado de Investigaciones y Posgrado, con la planificación de los resultados a nivel de la Cujae, a partir de un análisis de los listados o clasificaciones, los cuales se desagregan a nivel de facultad, con base en la cantidad de profesores equivalentes. 
incrementado en los últimos años, existiendo en la actualidad una gran variedad de listas a nivel internacional y regional; en muchas de las cuales se emplean indicadores bibliométricos, tanto de productividad científica (calculadas a partir del número de publicaciones indizadas), como de impacto (que incluye información acerca del número de citas bibliográficas derivadas de publicaciones científicas).

En esos listados no son visibles las contribuciones de las facultades que pertenecen a las universidades, sin embargo, dentro de las instituciones académicas esta información resulta de gran utilidad para contrastar los logros y avances de cada facultad y su aporte a los resultados obtenidos por la institución en conjunto.

De ahí la importancia de la evaluación de la producción científica de las IES a partir de estudios bibliométricos, lo cual ha adquirido especial relevancia en las últimas décadas. La bibliometría la han definido múltiples autores, entre ellos Arencibia y Araújo (2004), quienes expresaron que ésta ayuda a precisar, a través del estudio de las publicaciones, el desarrollo de una determinada área de la ciencia y que su alcance abarca desde la producción científica de un individuo, grupo o institución de investigación, hasta el comportamiento de todo un sector o campo temático durante un periodo dado y en un entorno nacional o internacional.

Los estudios bibliométricos aportan información (cuantitativa y cualitativa) sumamente valiosa, de apoyo para la toma de decisiones en materia de planificación y evaluación de las actividades de investigación en el ámbito universitario. Si se aplican esos estudios bibliométricos al análisis de las contribuciones científicas de las facultades de instituciones universitarias, permiten identificar las fortalezas de cada cual, así como revelar sus perfiles temáticos, las relaciones de cooperación científica entre sí, los patrones de colaboración nacional e internacional de cada uno de estos dominios de la ciencia, y su vinculación con el desarrollo de ésta en la universidad en su conjunto, del país u otros países e instituciones del mundo.

El objetivo de este artículo es representar y analizar el perfil investigador de la Facultad de Ingeniería Industrial de la Cujae en bases de datos de corriente principal, a partir de un estudio bibliométrico de la producción científica de sus investigadores durante el periodo 2003-2012.

Mediante la evaluación de una batería de indicadores bibliométricos validados internacionalmente, se analiza la dimensión cuantitativa y cualitativa de la producción científica de esta facultad, así como los patrones de colaboración nacional e internacional en los artículos, lo que permite ofrecer una imagen de la intensidad de la investigación en diversos niveles de agregación y determinar la visibilidad e impacto de estas publicaciones en el plano internacional. 
Su realización proporciona información valiosa sobre los resultados de la investigación de esta facultad, que circulan actualmente entre la comunidad científica. El procedimiento propuesto se emplearía para el estudio de las publicaciones científicas del resto de las facultades de la Cujae, con miras a obtener una imagen más completa del perfil investigativo de la institución, así como de otras pertenecientes a universidades cubanas y extranjeras. Además, proporciona una herramienta para la toma de decisiones en materia de política científica, de tal manera que contribuya a mejorar la posición de la institución en las clasificaciones de las universidades en el ámbito internacional.

\section{MÉTodos}

Tras una larga revisión de la literatura, se detectó un grupo de trabajos publicados desde 2000 hasta la fecha, en los que se aplica el análisis bibliométrico a la producción científica de las IES (principalmente universidades), los cuales se estudiaron con la finalidad de determinar los procedimientos seguidos, las fuentes de información empleadas, los sistemas utilizados para el procesamiento de la información, el periodo de análisis, los indicadores evaluados, entre otros elementos.

Algunos ejemplos de este tipo de estudios bibliométricos relativos a una institución, específicamente a las universidades, lo constituyen los trabajos de Kim y Kim (2000), en el Departamento de Química de la Universidad Nacional de Seul, Corea; Sanz et al. (2005), en la Facultad de Humanidades de la Universidad de Mar del Plata, Argentina; Alonso et al. (2006), con un estudio de dominio de la Universidad Politécnica de Valencia, España; Olmeda et al. (2008), que tratan sobre la producción científica de las universidades en la comunidad de Cataluña, y Kumar y Husain (2010), con un análisis bibliométrico de los resultados de investigación de la Universidad Jamia Millia Islamia de Nueva Delhi, en el campo de las ciencias naturales.

A nivel nacional se detectaron los trabajos de Martí (2007), donde se analiza la producción científica de la Universidad de La Habana; Peralta et al. (2011), quienes examinan la producción científica de la Universidad Central "Marta Abreu" de Las Villas, y la tesis doctoral de Arencibia (2010), en la que se aplica un grupo de indicadores bibliométricos validados internacionalmente, a la producción de la ciencia y la educación superior cubanas (Arencibia, 2010).

Se obtuvieron como aspectos comunes el uso de las bases de datos de la corriente principal, el empleo de software para el almacenamiento y análisis de la información, además de una estrategia similar de búsqueda, extracción 
Teniendo en cuenta estos resultados, se diseñó un procedimiento basado en las propuestas de varios autores y se incorporaron algunos elementos necesarios para la evaluación de la producción científica de los investigadores de una facultad universitaria, como el análisis de las clasificaciones universitarias, para que sirvan de referencia en el estudio bibliométrico.

El procedimiento diseñado se representa mediante un diagrama de flujo en la Figura 1, y a continuación se detallan cada una de las actividades que lo conforman.

Figura 1. Diagrama de flujo del procedimiento para la realización del estudio bibliométrico

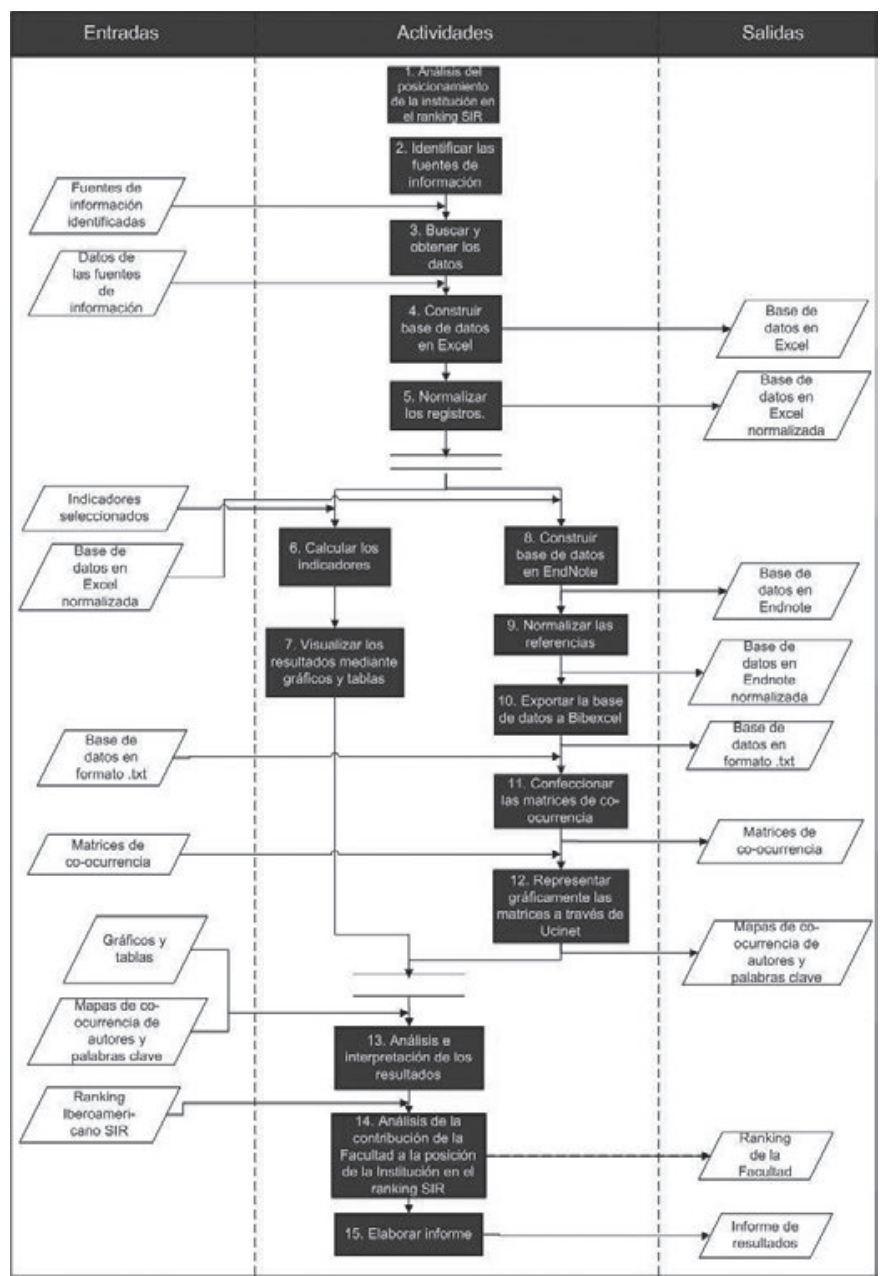

Fuente: elaboración propia. 


\section{Análisis del posicionamiento de la institución en el SIR iberoamericano}

Como se expresó anteriormente, las clasificaciones (o rankings) constituyen instrumentos que aportan información valiosa a las IES sobre el comportamiento de la producción de conocimientos, mediante la medición de indicadores comparativos. Específicamente el SIR (Scimago Institutions Ranking) iberoamericano, uno de los listados más reconocidos y aprobados por la comunidad científica internacional, evalúa indicadores bibliométricos que se basan fundamentalmente en la producción, la colaboración y el impacto.

Por ello inicia el estudio con el análisis de la posición de la institución en las ediciones de esta clasificación iberoamericana, la cual se deriva de los resultados de los indicadores anteriores, que a su vez se basan en las publicaciones científicas en revistas indizadas en la base de datos Scopus en diferentes periodos. Este análisis inicial resulta de gran utilidad, pues permite obtener una visión general del posicionamiento de la institución a nivel iberoamericano y la comparación con otros centros a nivel nacional.

\section{Selección del objeto de estudio}

Primeramente, se selecciona el objeto de estudio, que en este caso se basa en las publicaciones científicas en revistas indizadas en las bases de datos Scopus y WoS de los profesores de una facultad.

Esta decisión está en correspondencia con la tendencia internacional de evaluar el mérito científico, fundamentalmente sobre la base de publicaciones en revistas científicas, resultando que, en la mayoría de los estudios bibliométricos, los artículos son más frecuentemente la unidad de análisis seleccionada, pues son considerados altamente representativos del resultado real de toda actividad de investigación (Alonso et al., 2006; Olmeda et al., 2008).

Igualmente, se tuvo en cuenta que la publicación en revistas indizadas en fuentes internacionales, y especialmente en las bases de datos de corriente principal, además de permitir la visibilidad y el reconocimiento internacional de las contribuciones científicas, es un indicador de la calidad y prestigio de los resultados de investigación, lo cual — desde la perspectiva de los indicadores bibliométricos - es una manera de garantizar su validez y confiabilidad a la hora de comparar el perfil, patrones y posicionamiento científico de países e instituciones.

El periodo analizado abarca los últimos diez años, pues se considera un tiempo suficiente para que los resultados científicos publicados en bases de datos internacionales hayan generado un impacto a través de las citas recibidas. 
Además, en los estudios bibliométricos analizados, aproximadamente el 50\% de éstos abarca un periodo de 7 a 13 años.

\section{Identificación de las fuentes de información}

Las principales fuentes de información utilizadas son los sistemas de información científica de la institución, los cuales actualmente se perfilan como los candidatos idóneos para ser la fuente de información sobre la actividad científica de las universidades.

Diferentes bases de datos en línea se emplean para la recuperación de los artículos a texto completo o sus metadatos, como el Directory of Open Access Journals (DOAJ), Dialnet (Fundación de la Universidad de la Rioja, España), e-revistas (portal del Consejo Superior de Investigaciones Científicas-CSIC de España) y SciELO (Scientific Electronic Library Online), así como los sitios web de las revistas que se editan en formato electrónico.

Para el análisis de citas se emplea Google Scholar y la opción Author Preview disponible de forma gratuita en Scopus. Por último, los portales scimago Journal and Country Rank (SJCR) y sCImago Institutions Ranking (SIR), desarrollados por el Grupo scimago, se usan para obtener información bibliométrica basada en los datos que recoge Scopus.

\section{Aplicación de una estrategia de búsqueda, extracción y procesamiento de los datos primarios}

El objetivo de la búsqueda consiste en recuperar los artículos publicados por los investigadores de la facultad en las bases de datos Scopus y WoS en los últimos diez años.

Para ello se debe acceder a los sistemas de información científica de la institución, donde se registran sus resultados de investigación científica. Posteriormente, se deben realizar búsquedas en las bases de datos en línea y en los sitios web de las revistas, con el fin de recuperar estos documentos a texto completo (en los casos en que sea posible el acceso) y sus metadatos, lo que permite corroborar los datos reportados y completar los registros incompletos.

Los datos se almacenan en una base de datos con toda la información integrada mediante Microsoft Excel, la cual incluye los campos siguientes: año de registro en el balance, título, idioma, autores y para cada autor, categoría científica, afiliación y sector al que pertenece; tipo de colaboración, grupo temático, año de publicación, nombre de la revista, ISSN/eISSN, país, volumen, número, páginas, palabras clave, resumen, bases de datos y URL. 
Posterior a este proceso, se desarrolla la normalización (control de errores) de los campos directamente relacionados con los distintos niveles de análisis (instituciones, revistas, autores, etc.). Este último requiere de un riguroso trabajo manual dentro de la base de datos, porque con frecuencia no es uniforme la denominación de la institución ni de las facultades y centros en el campo "Afiliación". Se identifican y normalizan los nombres de los autores de los trabajos, confrontando los datos con las listas de la plantilla de docentes e investigadores de la facultad, para los autores pertenecientes actualmente a la institución, y realizando consultas en diferentes bases de datos y otras fuentes disponibles a través de Internet para el resto de los autores.

Además, se clasifican los registros según el tipo de colaboración identificada en los artículos, la procedencia de cada autor (especificando sector, institución, provincia y país) y el grupo temático, a partir la clasificación de temáticas definidas por la revista Ingeniería Industrial.

Toda la información de los artículos se introduce de manera semiautomática en una base de datos bibliográfica, empleando el gestor de referencias EndNote X4, con el objetivo de crear mapas de co-ocurrencia de palabras claves y autores, con la ayuda de los softwares Bibexcel 1.0 y Ucinet 6.123. Los análisis estadísticos como el de series cronológicas, para conocer cómo han evolucionado en el tiempo las variables estudiadas, se realizan con la ayuda del software Minitab 14.

Para el análisis del impacto esperado de la producción científica, se descargan las hojas de datos de Microsoft Excel disponibles en el SJCR, con toda la información bibliométrica existente en el portal sobre las revistas indizadas en Scopus en las que publicaron los investigadores analizados.

Para contabilizar las citas recibidas y determinar el impacto real alcanzado por las publicaciones de cada investigador, se realizan búsquedas de los artículos y los autores en Scopus y Google Scholar, empleando de forma aleatoria los términos nombre y apellidos del autor, título del artículo y revista en la que se publicó; hasta conseguir la ecuación que mejores resultados aporte.

Por último, se accede a los reportes del SIR iberoamericano, con vistas a extraer la información relativa a la posición y los indicadores de las IES a las que están afiliados los autores que publican en colaboración con los de la facultad.

\section{Cálculo de los indicadores bibliométricos. Visualización, análisis e interpretación de los resultados}

Tomando como base los indicadores evaluados en la más reciente edición del SIR y luego del análisis de los estudios bibliométricos de dominio institucional ya mencionados, en los que se constató la utilización de diferentes 
indicadores, técnicas y métodos estadísticos para el análisis de la información, se dedicó especial énfasis en esta investigación en una selección de la batería de indicadores bibliométricos propuestos por Arencibia (2010), en correspondencia con los utilizados en otros estudios nacionales e internacionales, destinados a capturar la dimensión cuantitativa y cualitativa de la producción científica nacional; y para la caracterización de los diversos tipos de colaboración en los artículos (Arencibia, 2010).

A continuación, se mencionan los indicadores seleccionados y su relación con los utilizados en el sIR iberoamericano de 2013.

\section{Indicadores para la dimensión cuantitativa de la producción científica}

Número de artículos o Producción total (A), Proporción de artículos respecto del total (\% A), Tasa de Variación (TV), Ratio A-Investigador y Ratio A$\mathrm{PhD}$ (Arencibia, 2010).

El indicador A, que señala el número total de artículos que se estudian, sea cual fuere el nivel de agregación analizado (Arencibia, 2010), equivale al evaluado en el listado denominado Producción Cientifica (O, por Output), referido al número total de documentos publicados en revistas académicas indizadas en Scopus (SRG, 2013).

\section{Indicadores para la colaboración científica}

Número de artículos en colaboración (A-col), Artículos sin colaboración (SC), Artículos con colaboración nacional exclusiva (CN), Artículos con colaboración nacional e internacional (CNI), Artículos con colaboración internacional (CI), Producción científica con colaboración internacional (PCI), Producción científica nacional exclusiva (PNE) y Tasas de colaboración (\% CI, CNI, CN, SC, A-col, PCI y PNE) (Arencibia, 2010). A éstos se adicionó el Índice de co-autoría (IC), que aunque no es evaluado por Arencibia (2010), lo utiliza la mayor parte de los autores consultados.

Dentro de este grupo, el indicador\% PCI, que recoge la proporción de artículos publicados en los que al menos un autor pertenece a una institución internacional, se corresponde con Colaboración Internacional (\% IC, por International Collaboration) empleado en la clasificación (SRG, 2013).

Se relaciona también con este grupo el indicador Liderazgo Científico (\% Lead, por Scientific Leadership), presente en la clasificación a partir de la edición de 2013, que se define como la producción de una institución en la que ésta es "el principal contribuidor", es decir, el número de trabajos en los que el "corresponding author" pertenece a la institución (SRG, 2013). 


\section{Indicadores para la dimensión cualitativa de la producción científica}

Distribución por cuartiles (Q1, Q2, Q3, Q4), Número de artículos de alta visibilidad (AQ1), Proporción de artículos de alta visibilidad (PAQ1), Número total de artículos citados (AC), Proporción de artículos citados (\% AC), Número total de citas recibidas (C), Promedio de citas por artículo (C/A), Índice $\mathrm{H}(\mathrm{i}-\mathrm{H})$ (Arencibia, 2010).

Específicamente el indicador PAQ1, equivale a Publicaciones de Alta Calidad (\% Q1, por High Quality Publications), definido en el listado como la relación de documentos que publica una institución en las revistas con más influencia del mundo, las ubicadas en el primer cuartil (25\%) de categoría, según la ordenación derivada del indicador scimago (SRG, 2013).

Mientras que la Ratio de Excelencia (\% Exc, por Excellence Rate), indica la cantidad (en porcentaje) de producción científica de una institución que se ha incluido en el grupo del 10\% de trabajos más citados de su campo científico. Es una medida de la cantidad de producción científica de alta calidad de las instituciones de investigación (SRG, 2013).

Este indicador toma como base para su cálculo la cantidad de citas recibidas por cada artículo, por lo cual se relaciona con el Número total de citas recibidas (C), definido por Arencibia (2010) como la sumatoria de las citas recibidas por cada artículo citado.

Por último, el Impacto Normalizado (NI, por Normalized Impact), se calcula de la siguiente forma: los valores (en\%) muestran las relaciones entre el impacto científico medio de una institución y el conjunto promedio mundial con una puntuación de 1 , es decir, una puntuación de NI de 0.8 significa que la institución es citada un $20 \%$ por debajo del promedio mundial y un valor de 1.3 significa que la institución es citada un $30 \%$ superior a la media del mundo (SRG, 2013).

Igualmente, este indicador toma como base para su cálculo el Promedio de citas por artículo (C/A), definido por Arencibia como la media de citas recibidas por el conjunto de la producción científica, campo temático o cualquier otro nivel de agregación, que indica de forma directa el impacto o visibilidad alcanzada por un grupo de artículos (Arencibia, 2010).

Los resultados de los indicadores bibliométricos calculados se visualizan mediante cuadros y figuras, los cuales permiten un mejor análisis e interpretación. 


\section{Análisis de la contribución de la Facultad a la posición de la institución en el SIR iberoamericano}

Una vez realizado el estudio bibliométrico que permite analizar en detalle la producción científica de los investigadores de la facultad, conocer el grado de visibilidad e impacto que ha alcanzado a nivel mundial y caracterizar sus patrones de colaboración; se determina la contribución de la facultad a la posición de la institución en el SIR iberoamericano, a través de la evaluación de los indicadores de dicho listado, utilizando las publicaciones científicas en revistas indizadas en Scopus por la facultad, en los periodos analizados en sus distintas ediciones, así como su comparación con los valores obtenidos por la universidad.

Esto permite concluir sobre las fortalezas y debilidades de la actividad investigativa de la facultad y proporciona información útil para su gestión, con vistas al aumento continuo de la visibilidad e impacto de las publicaciones de sus investigadores, y al mejoramiento de la posición de la institución en las clasificaciones. Así, se evidencia la concepción del procedimiento como un ciclo de mejora continua.

\section{Elaboración del informe de resultados del estudio y su presentación}

Los resultados obtenidos mediante este estudio bibliométrico se presentan en un informe y se comunican a los investigadores, preferiblemente mediante seminarios dirigidos a los grupos de investigación, de tal forma que se genere el intercambio de conocimientos y se socialicen las prácticas de los investigadores que probaron dar resultados positivos, en relación con la publicación de artículos científicos en las bases de datos de corriente principal.

\section{Resultados}

A continuación, se exponen los resultados obtenidos mediante la aplicación del procedimiento para la realización del estudio bibliométrico de la producción científica en bases de datos de corriente principal diseñado a la producción científica de la Facultad de Ingeniería Industrial de la Cujae en el periodo 2003-2012.

\section{Análisis de la posición de la institución en el SIR iberoamericano}

En los resultados reportados en el listado del SIR 2010, 2011, 2012 y 2013, se observa que a pesar de que la Cujae ha mostrado un comportamiento ligeramente 
creciente (en cuanto a la cantidad de artículos), no se ha desempeñado a la par con la producción de las otras universidades, lo que se refleja en el descenso de su posición en los reportes de 2010 a 2012, aunque en el del 2013 comienza a mejorar.

En el SIR iberoamericano 2013, que incluye toda la producción científica presente en la base de datos Scopus en el periodo 2007-2011, se pudo conocer que de más de 1,600 universidades iberoamericanas, la Cujae ocupó la posición 306 con 205 artículos, precedida a nivel nacional por la Universidad de La Habana, que se encuentra en la posición 122 con 1,386 artículos, además del Instituto Superior de Ciencias Médicas de La Habana, la Universidad Central "Marta Abreu" de Las Villas y la Universidad de Oriente.

En cuanto al resto de los indicadores que evalúa este listado, cabe concluir que el porcentaje de publicaciones científicas de la Cujae, elaboradas conjuntamente con instituciones de otros países, se ha mantenido entre los más altos con relación con el resto de las universidades cubanas y ha aumentado ligeramente en los últimos años, alcanzando el 78.54\% en el 2013.

Por otra parte, el Liderazgo Científico se encuentra entre los más bajos, en relación con otras instituciones cubanas (33.17\%). En cuanto a la proporción de publicaciones en revistas del primer cuartil, ésta ha descendido en el último periodo y sus valores no rebasan el 50\%. Por último, los indicadores que miden el impacto científico (Ratio de Excelencia e Impacto Normalizado), se han mantenido con niveles bajos en relación con el comportamiento internacional, pero similares a los resultados de las instituciones cubanas.

\section{Selección del objeto de estudio}

El objeto de estudio lo constituyen las publicaciones científicas de los profesores de la Facultad de Ingeniería Industrial en las bases de datos Scopus y Web of Science durante el periodo 2003-2012.

\section{Identificación de las fuentes de información. Aplicación de una estrategia de búsqueda, extracción y procesamiento de los datos primarios}

Las principales fuentes de información utilizadas durante la investigación fueron, en primer lugar, el Sistema de Registros Primarios de la Cujae y los Informes de Balance Anuales de Investigaciones y Posgrado de la Facultad de Ingeniería Industrial emitidos en el periodo 2003-2012. Además, se emplearon las diferentes bases de datos en línea ya mencionadas, Google Scholar y Scopus para el análisis de citas, así como los portales del Grupo scimago. A partir de las fuentes de información señaladas, se siguió la 
estrategia de búsqueda, extracción y procesamiento de los datos descrita en la sección anterior.

\section{Cálculo de los indicadores bibliométricos. Visualización, análisis e interpretación de los resultados}

Los resultados obtenidos por cada grupo de indicadores se presentan enseguida.

\section{Indicadores para la dimensión cuantitativa de la producción cientifica}

El total de artículos en revistas de corriente principal fue 48. Su distribución por año de publicación se muestra en la Figura 2, en la que se aprecia una tendencia al crecimiento, la cual se ajusta mejor a un modelo cuadrático, ya que los valores muestran un comportamiento ligeramente creciente en los primeros años del periodo estudiado y luego, su ritmo su crecimiento es mucho más acelerado.

Figura 2. Distribución del número de artículos por año de publicación en el periodo 2003-2012

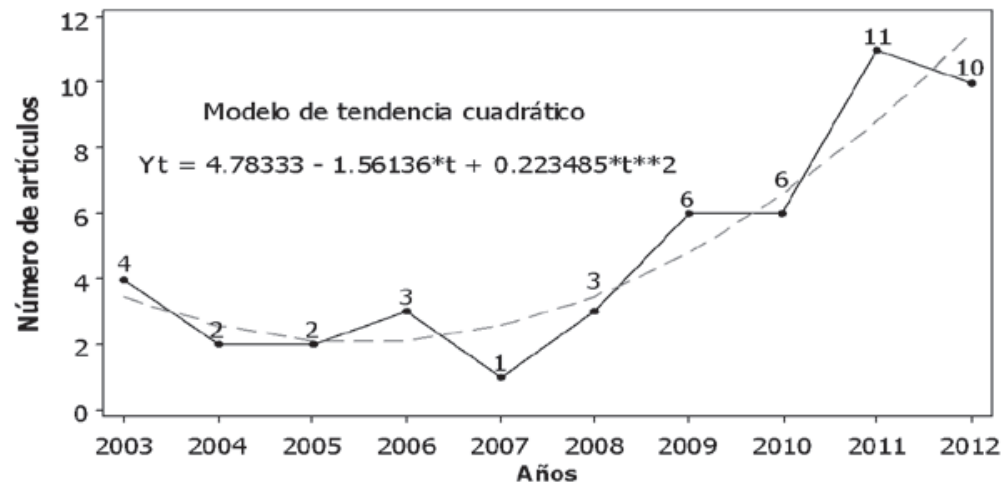

Fuente: elaboración propia, a partir de las fuentes de información descritas en el epígrafe 2.

Para determinar las diferencias entre la cantidad de artículos publicados en un año respecto del anterior, en términos porcentuales, se calculó la tasa de variación anual, lo cual se representa en la Figura 3. 
Figura 3. Tasas de variación en la producción de artículos de la Facultad de Ingeniería Industrial en el periodo 2003-2012

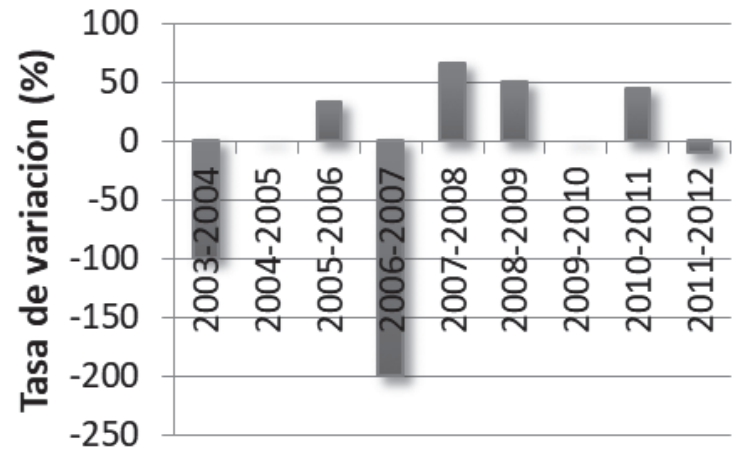

Fuente: elaboración propia, a partir de las fuentes de información descritas en el epígrafe 2.

Las tasas de variación anual fueron significativas, siendo los periodos 2003-2004 y 2006-2007 los de mayor variabilidad en sentido negativo. La tasa de variación total en el periodo 2003-2012, fue de 60\%.

El análisis de la producción por sectores reveló que la mayoría de los artículos fueron publicados en colaboración con autores pertenecientes a instituciones de educación superior y, en menor medida, a centros de investigación y empresas, como se muestra en la Figura 4.

Figura 4. Distribución de la producción científica por sectores en el periodo 2003-2012

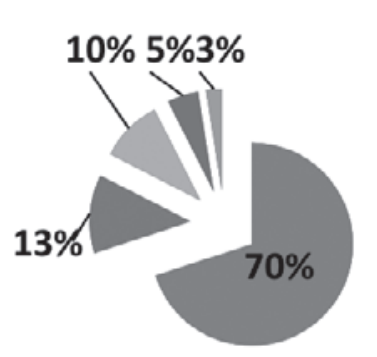

\section{Sectores}

\section{ES - Educación}

Superior (sin

Cujae)

CT - Ciencia y

Técnica

EM - Empresa

口OT - Otros

Fuente: elaboración propia, a partir de las fuentes de información descritas en el epígrafe 2.

Debe tenerse en cuenta que se han empleado datos a partir del recuento completo de coautorías, según el cual se atribuye la autoría a todas y cada una de las instituciones firmantes, lo cual provoca solapamientos en los recuentos totales de producción al atribuirse cada trabajo a tantos autores co- 
El estudio de la producción por países mostró que los investigadores de la facultad publicaron en el periodo en mayor grado con autores pertenecientes a instituciones nacionales $(37 \%)$ y de las extranjeras, se destacan por su producción las provenientes de España y México, lo que se observa en la Figura 5.

Figura 5. Distribución de la producción científica por países en el periodo 2003-2012

\begin{tabular}{|c|c|c|}
\hline Países colaboradores & Número de artículos (A) & $\% \mathbf{A}$ \\
\hline Cuba & 11 & 37 \\
\hline España & 8 & 27 \\
\hline México & 7 & 23 \\
\hline Brasil & 5 & 17 \\
\hline Argentina & 4 & 13 \\
\hline Alemania & 3 & 10 \\
\hline Colombia & 2 & 7 \\
\hline Uruguay & 1 & 3 \\
\hline Total & 30 & 100 \\
\hline
\end{tabular}

Fuente: elaboración propia, a partir de las fuentes de información descritas en el epígrafe 2.

A nivel nacional, la producción en colaboración estuvo marcada por la provincia de La Habana, mientras que sólo dos artículos fueron publicados en conjunto con autores de otras regiones del país, como Matanzas y Pinar del Río, según se evidencia en la Figura 6, lo cual se explica por la cercanía geográfica.

Figura 6. Distribución de la producción científica por provincias cubanas en el periodo 2003-2012

\begin{tabular}{|c|c|c|}
\hline Provincia & Número de artículos (A) & $\% \mathbf{A}$ \\
\hline HAB & 9 & 81.8 \\
\hline MTZ & 1 & 9.1 \\
\hline PRI & 1 & 9.1 \\
\hline Total & 11 & 100 \\
\hline
\end{tabular}

Fuente: elaboración propia, a partir de las fuentes de información descritas en el epígrafe 2.

Es notable el predominio del idioma español, representando el 56\% del total de artículos, siendo el porcentaje de documentos en inglés de sólo un $40 \%$ y el $4 \%$ restante en portugués. En la Figura 7 se representa la distribución de los artículos por temáticas, en la que se observa que el mayor 
porcentaje lo representan las publicaciones que abordan la Gestión del Conocimiento, en muchos casos en conjunto con temas de Gestión de Recursos Humanos, Dirección e Informática Empresarial.

Figura 7. Distribución de la producción científica por temáticas en el periodo 2003-2012

\section{Temáticas}

= Gestión del conocimiento

E Informática Empresarial

Estadistica

nestión de Recursos

Humanos

- Investigación de Operaciones

- Dirección
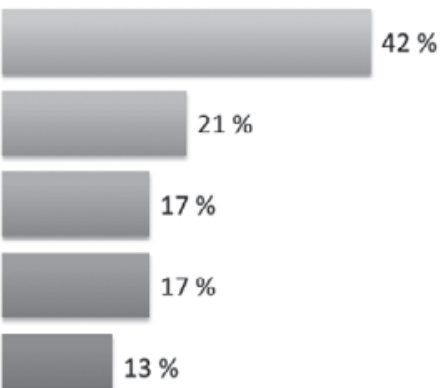

$13 \%$

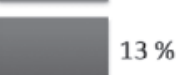

- Logística

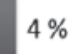

- Ergonomía

Fuente: elaboración propia, a partir de las fuentes de información descritas en el epígrafe 2.

A partir del número de trabajos publicados por autor, se distribuyeron los investigadores de la facultad en tres niveles de productividad: pequeños productores, que son aquellos autores que han firmado entre 1 y 4 trabajos; los medianos productores, que cubren una producción entre 5 y 10 trabajos; y los grandes productores, que son los que firmaron más de 10 trabajos. En la Figura 8 se aprecia cómo un número reducido de autores (1 gran productor y 3 medianos productores) publicó casi la mitad de los trabajos (44\%), mientras que sólo un investigador aportó más de diez artículos. 
VISIBILIDAD E IMPACTO DE LA PRODUCCIÓN CIENTÍFICA DE LA FACULTAD DE INGENIERÍA. .

Figura 8. Distribución de autores según niveles de productividad en el periodo 2003-2012

\begin{tabular}{|c|c|c|c|c|c|}
\hline $\begin{array}{c}\text { Niveles de } \\
\text { productividad }\end{array}$ & $\begin{array}{c}\text { Rango de } \\
\text { artículos }\end{array}$ & $\begin{array}{c}\text { Número de } \\
\text { artículos } \\
\text { (A) }\end{array}$ & $\%$ A & $\begin{array}{c}\text { Número } \\
\text { de autores } \\
\text { Facultad }\end{array}$ & $\begin{array}{c}\text { \% autores } \\
\text { Facultad }\end{array}$ \\
\hline $\begin{array}{c}\text { Pequeños } \\
\text { productores }\end{array}$ & $1-4$ & 35 & 56 & 25 & 86 \\
\hline $\begin{array}{c}\text { Medianos } \\
\text { productores }\end{array}$ & $5-10$ & 16 & 25 & 3 & 10 \\
\hline Grandes productores & Más de 10 & 12 & 19 & 1 & 3 \\
\hline & Total & 63 & 100 & 29 & 100 \\
\hline
\end{tabular}

Fuente: elaboración propia, a partir de las fuentes de información descritas en el epígrafe 2.

Se contabilizó, además, el número de autores que produjeron un solo artículo, resultando este valor el 59\% del total de autores de la Facultad que tienen publicaciones en revistas indizadas en bases de datos de corriente principal.

En promedio, se han publicado en el periodo estudiado en revistas de corriente principal, 0.44 artículos por profesor (48 trabajos entre 108 profesores que trabajaron como promedio en el periodo 2003-2012 en la facultad) y 1.26 artículos por doctor (48 trabajos entre 38 doctores como promedio en el periodo).

\section{Indicadores para la colaboración cientifica}

En las 48 contribuciones de la facultad en Scopus y la Web of Science, participaron 78 autores diferentes con un total de 144 firmas. El índice de coautoría es de 3 autores por artículo, con una variación entre 2 y 4 para todo el periodo estudiado.

Como se observa en el Cuadro 1, la mayoría de los artículos analizados fueron elaborados en colaboración (A-col), siendo superior la proporción de artículos con colaboración internacional (CI) con relación a la nacional (CN).

Cuadro 1. Indicadores de colaboración en el periodo 2003-2012

\begin{tabular}{|l|c|c|}
\hline \multicolumn{1}{|c|}{ Indicador } & Total & Tasa de colaboración \\
\hline Número de artículos en colaboración (A-col) & 32 & $67 \%$ \\
\hline Artículos sin colaboración (SC) & 16 & $33 \%$ \\
\hline Artículos con colaboración nacional exclusiva (CN) & 8 & $17 \%$ \\
\hline
\end{tabular}




\begin{tabular}{|l|c|c|}
\hline \multicolumn{1}{|c|}{ Indicador } & Total & Tasa de colaboración \\
\hline Artículos con colaboración nacional e internacional (CNI) & 3 & $6 \%$ \\
\hline Artículos con colaboración internacional (CI) & 21 & $44 \%$ \\
\hline Producción científica con colaboración internacional (PCI) & 24 & $50 \%$ \\
\hline Producción científica nacional exclusiva (PNE) & 24 & $50 \%$ \\
\hline Artículos en los que el primer autor pertenece a la facultad & 26 & $54 \%$ \\
\hline
\end{tabular}

Fuente: elaboración propia, a partir de las fuentes de información descritas en el epígrafe 2.

$\mathrm{Al}$ analizar la tendencia de las tasas de colaboración nacional (\% CN) y de producción con colaboración internacional (\% PCI), se evidencia que en todos los años el número total de artículos publicados, en los que al menos un autor pertenece a una institución internacional, ha superado a los trabajos de autores afiliados a instituciones nacionales, como se muestra en la Figura 9.

Figura 9. Tasas de colaboración de PCI y CN en el periodo 2003-2012

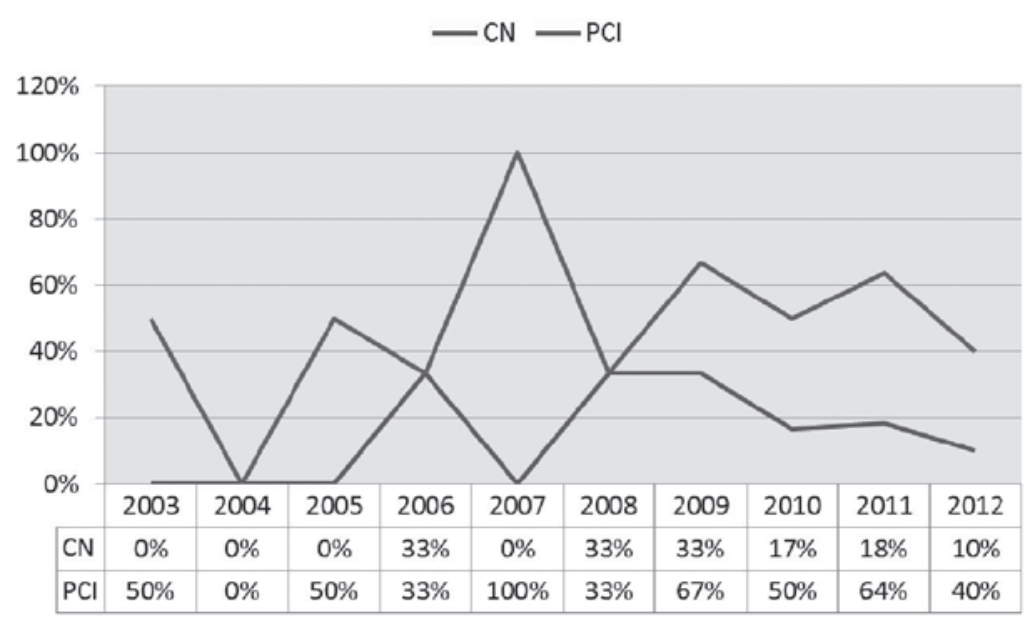

Fuente: elaboración propia a partir de las fuentes de información descritas en el epígrafe 2.

En un estudio más detallado, se analizaron las instituciones universitarias de procedencia de los autores que publicaron con la facultad, las cuales se muestran en el Cuadro 2, junto a sus posiciones en el SIR iberoamericano 2013. 
VISIBILIDAD E IMPACTO DE LA PRODUCCIÓN CIENTÍFICA DE LA FACULTAD DE INGENIERÍA..

Cuadro 2. Universidades con las que la Facultad de Ingeniería Industrial colabora y sus posiciones en el SIR iberoamericano 2013

\begin{tabular}{|c|c|c|c|c|}
\hline \multirow{2}{*}{ Universidades } & \multirow{2}{*}{ País } & \multirow{2}{*}{ Frecuencia } & \multicolumn{2}{|c|}{ Posición en el SIR 2013} \\
\hline & & & Iberoamérica & Latinoamérica \\
\hline Universidad de Valladolid & España & 8 & 55 & - \\
\hline Instituto Politécnico Nacional & México & 6 & 36 & 16 \\
\hline Universidad Nacional de Mar del Plata & Argentina & 6 & 99 & 49 \\
\hline Universidad de La Habana & Cuba & 4 & 122 & 67 \\
\hline Universidade Catolica de Brasilia & Brasil & 4 & 180 & 117 \\
\hline Universidade do Vale do Rio dos Sinos & Brasil & 4 & 182 & 119 \\
\hline Carl von Ossietzky University & Alemania & 3 & * & * \\
\hline Universidad de Sevilla & España & 3 & 20 & - \\
\hline Universidad Autónoma de Sinaloa & México & 3 & 224 & 153 \\
\hline $\begin{array}{l}\text { Instituto Superior de Tecnologías y } \\
\text { Ciencias Aplicadas }\end{array}$ & Cuba & 3 & 348 & 267 \\
\hline Universidad de la República & Uruguay & 2 & 69 & 32 \\
\hline Universidad Nacional de San Juan & Argentina & 2 & 193 & 126 \\
\hline Universidad Autónoma de Madrid & España & 1 & 13 & - \\
\hline Universidad de Oviedo & España & 1 & 34 & - \\
\hline Universidad Autónoma de Nuevo León & México & 1 & 96 & 47 \\
\hline Universidad de Sonora & México & 1 & 165 & 105 \\
\hline $\begin{array}{l}\text { Universidad de Matanzas "Camilo Ci- } \\
\text { enfuegos" }\end{array}$ & Cuba & 1 & 353 & 272 \\
\hline $\begin{array}{l}\text { Universidad de Pinar del Río Hermanos } \\
\text { Saíz Montes de Oca }\end{array}$ & Cuba & 1 & 384 & 302 \\
\hline Universidad de San Buenaventura & Colombia & 1 & 389 & 307 \\
\hline Universidad Libre & Colombia & 1 & 411 & 328 \\
\hline
\end{tabular}

* Nota: Los datos de la Carl von Ossietzky University de Alemania, no pudieron obtenerse ya que la clasificación analizada sólo toma en cuenta a los países de Iberoamérica.

Fuente: elaboración propia, a partir de datos del SIR 2012.

La colaboración entre facultades, centros y direcciones de la Cujae sólo se manifestó en tres publicaciones, en las que participaron dos autores de la Facultad de Ingeniería Informática. Por otra parte, la colaboración entre los departamentos de la Facultad de Ingeniería Industrial sólo estuvo presente en cuatro artículos, siendo la más frecuente la existente entre el Departamento de Ingeniería Industrial y el extinto Departamento de Matemática General. 
En la Figura 10 se representa la red de colaboración de los autores de la facultad. Para la construcción de este mapa, se incluyeron todos los autores y se utilizaron diferentes colores para representar las temáticas en las que publican los profesores de la facultad con mayor frecuencia, así como para diferenciar a los autores externos.

Figura 10. Principales relaciones de colaboración de los autores

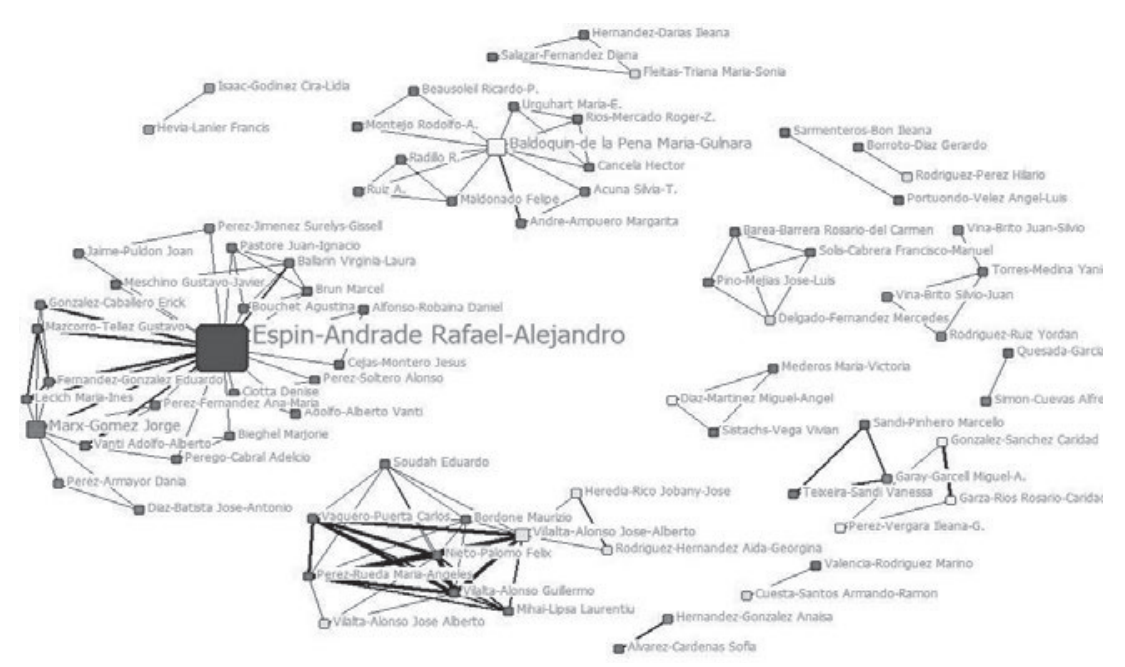

Fuente: elaboración propia, a partir de las fuentes de información descritas en el epígrafe 2.

El grado nodal se indica en la gráfica con un grosor más intenso de las líneas, reflejando así las categorías que co-ocurren con mayor frecuencia. El grado de intermediación se representa a través del tamaño de los nodos y de la fuente utilizada en el nombre de cada autor.

En este caso, se observa una red conformada por 14 subgrupos, que representan grupos de autores conectados directamente entre sí. Se le atribuye un mayor grado de intermediación al profesor Rafael Alejandro Espín-Andrade, que es el autor que mayor producción realizó en colaboración con autores externos, principalmente extranjeros, y con algunos autores de la facultad que investigan en las temáticas de Dirección e Informática Empresarial.

Otro de los clusters que más se destaca por su grado de intermediación es el ubicado en la parte superior izquierda de la red global, correspondiente a María Gulnara Baldoquín de la Peña, con publicaciones en el área de Investigación de Operaciones, en colaboración con autores externos. Además, en 
la zona inferior izquierda se ubica un cluster más concentrado y con relaciones más intensas, representando en su mayoría la actividad investigativa de la temática Estadística, con José Alberto Vilalta-Alonso como el profesor de mayor número de artículos.

Igualmente se realizó un examen de las palabras clave más utilizadas y se confeccionó la red de co-ocurrencia de los términos que se expone en la Figura 11. En esta red, tanto el tamaño de los nodos, como el color y la fuente utilizada, representan el grado de intermediación.

Como se aprecia, el nodo central, "lógica difusa", es el de mayor poder, según su grado de intermediación, el cual atrae hacia sí al nodo "lógica difusa compensatoria", con el que mantiene una relación muy estrecha, así como a un grupo numeroso de categorías que co-ocurren en los artículos que tratan estas temáticas (como "dirección estratégica", "toma de decisiones", "sistemas de información complejos", etcétera).

Figura 11. Análisis de co-ocurrencia de las palabras claves

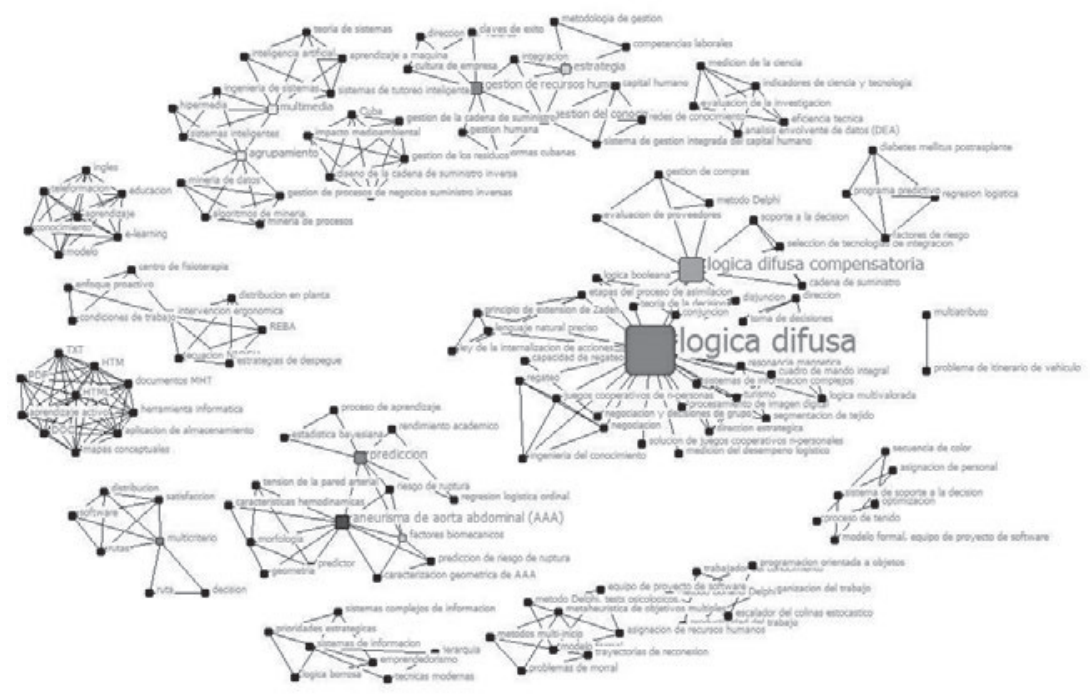

Fuente: elaboración propia, a partir de las fuentes de información descritas en el epígrafe 2.

Hacia la zona inferior izquierda se aprecia otra red, cuyo núcleo es el término "aneurisma de aorta abdominal (AAA)", presente en 4 artículos sobre Estadística, con el que se conectan categorías como "predicción", "caracterización geométrica de AAA", entre otros. 
Por último, se confeccionó un mapa de las relaciones de colaboración entre las instituciones a las que están afiliados los autores (Figura 12), el cual permitió mostrar a la Universidad de Valladolid y a AINIA Centro Tecnológico, ambos de España, como los nodos con mayor cercanía a la Cujae en la red, tanto a partir del grado nodal (expresado en el grosor de las líneas), como en la cercanía al nodo central.

Figura 12. Principales relaciones de colaboración entre las instituciones

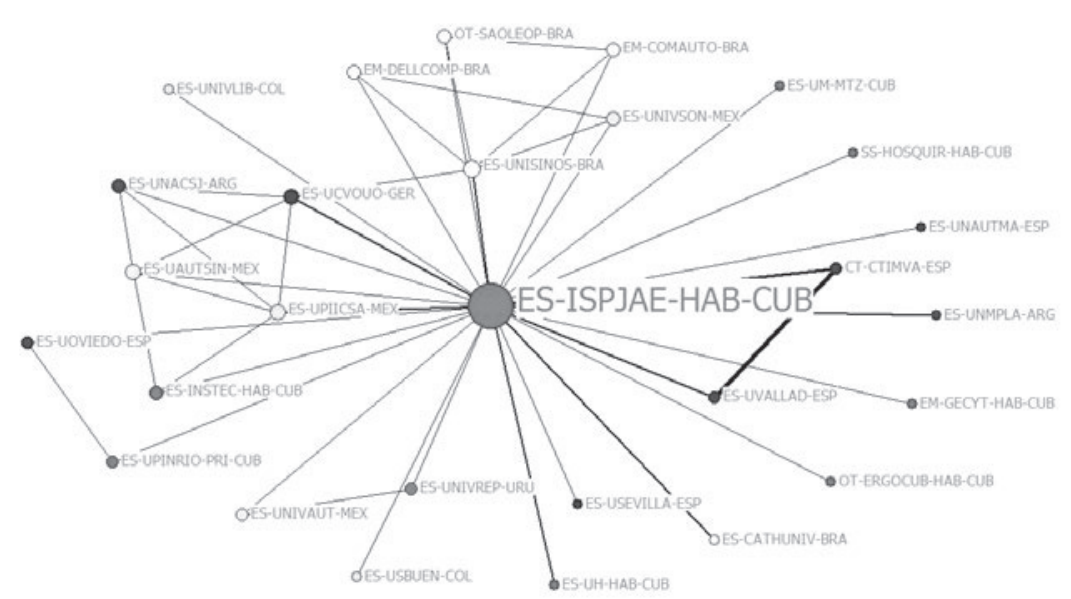

Fuente: elaboración propia, a partir de las fuentes de información descritas en el epígrafe 2.

\section{Indicadores para la dimensión cualitativa de la producción científica}

La producción científica estudiada fue publicada en un total de 29 revistas científicas, las cuales pertenecen en su mayoría a Holanda, Venezuela y Estados Unidos (Figura 13). El mayor número de artículos se publicó en revistas de Cuba y aproximadamente el $71 \%$ del total de trabajos pertenece a revistas extranjeras. 
VISIBILIDAD E IMPACTO DE LA PRODUCCIÓN CIENTÍFICA DE LA FACULTAD DE INGENIERÍA. .

Figura 13. Distribución de las revistas por países

\begin{tabular}{|l|c|c|c|c|}
\hline \multicolumn{1}{|c|}{ País } & Número de artículos (A) & $\% \mathbf{A}$ & $\begin{array}{c}\text { Número de revistas } \\
\text { (NR) }\end{array}$ & $\%$ NR \\
\hline Holanda & 8 & 17 & 5 & $17 \%$ \\
\hline Estados Unidos & 5 & 10 & 5 & $17 \%$ \\
\hline Venezuela & 5 & 10 & 4 & $14 \%$ \\
\hline España & 5 & 10 & 3 & $10 \%$ \\
\hline Inglaterra & 3 & 6 & 3 & $10 \%$ \\
\hline Cuba & 14 & 29 & 2 & $7 \%$ \\
\hline Brasil & 2 & 4 & 2 & $7 \%$ \\
\hline Rusia & 2 & 4 & 1 & $3 \%$ \\
\hline Alemania & 1 & 2 & 2 & $3 \%$ \\
\hline Colombia & 1 & 2 & 1 & $3 \%$ \\
\hline Costa Rica & 1 & 2 & 1 & $3 \%$ \\
\hline Portugal & 48 & 2 & 1 & $3 \%$ \\
\hline Total & 100 & 29 & 1 \\
\hline
\end{tabular}

Fuente: elaboración propia, a partir de las fuentes de información descritas en el epígrafe 2.

En el Cuadro 3 se relacionan las revistas en que publicaron los investigadores de la facultad con mayor frecuencia, donde se aprecia que la revista Investigación Operacional, indizada en Scopus, fue la más utilizada (con 13 artículos). El grupo formado por las seis revistas más productivas, que representan el 21\% del total, contienen el 50\% de los trabajos. El 79\% restante de los títulos de revistas identificados recogen un único artículo.

Cuadro 3. Revistas de corrientes principal utilizadas por los investigadores de la Facultad de Ingeniería Industrial para publicar artículos en el periodo 2003-2012

\begin{tabular}{|l|c|c|c|c|}
\hline \multicolumn{1}{|c|}{ Revista } & ISSN/eISSN & País & Base de datos & Frecuencia \\
\hline 1. Investigación Operacional & $0257-4306$ & Cuba & Scopus & 13 \\
\hline 2. DYNA & $0012-7361 / 1989-1490$ & España & $\begin{array}{c}\text { Web of Science } \\
\text { (SCIEX) }\end{array}$ & 3 \\
\hline 3. Journal of Biomechanics & $0021-9290 / 1873-2380$ & Inglaterra & $\begin{array}{l}\text { Web of Science } \\
\text { (SCIEX), Scopus }\end{array}$ & 2 \\
\hline 4. Work & $1051-9815 / 1875-9270$ & Países Bajos & $\begin{array}{c}\text { Web of Science } \\
\text { (SSCI), Scopus }\end{array}$ & 2 \\
\hline
\end{tabular}




\begin{tabular}{|l|c|c|c|c|}
\hline \multicolumn{1}{|c|}{ Revista } & ISSN/eISSN & País & Base de datos & Frecuencia \\
\hline $\begin{array}{l}\text { 5. Annals of Operations } \\
\text { Research }\end{array}$ & $0254-5330 / 1572-9338$ & Países Bajos & $\begin{array}{l}\text { Web of Science } \\
\text { (sciex), Scopus }\end{array}$ & 2 \\
\hline $\begin{array}{l}\text { 6. Revista Venezolana de } \\
\text { Gerencia }\end{array}$ & $1315-9984$ & Venezuela & $\begin{array}{l}\text { Web of Science } \\
\text { (ssci), Scopus }\end{array}$ & 2 \\
\hline
\end{tabular}

Nota: las abreviaturas empleadas corresponden a las siglas en inglés de las bases de datos de la Web of Science: Science Citation Index Expandex (SCIEX) y Social Science Citation Index (SSCI).

Fuente: elaboración propia, a partir de las fuentes de información descritas en el epígrafe 2.

Pertenecen a la Web of Science 19 revistas (ya sea al Science Citation Index Expandex o al Social Science Citation Index) con un total de 27 artículos. En Scopus se incluyen 23 revistas con 39 trabajos. En ambas bases de datos se encuentran 14 revistas y 18 documentos.

El análisis de la distribución por cuartiles para los últimos diez años, sólo fue posible realizarlo para las revistas indizadas en la base de datos Scopus, pues la Web of Science es una herramienta propietaria a la cual no se tuvo acceso. Como se muestra en el Cuadro 4, en el primer cuartil, el de mayor visibilidad, se encuentran 6 artículos, que constituyen el 15\% de los publicados en revistas de esta base de datos.

Cuadro 4. Distribución por cuartiles de los artículos publicados en revistas de Scopus

\begin{tabular}{|l|c|c|c|c|c|c|}
\hline \multirow{2}{*}{ Año } & \multicolumn{5}{|c|}{ Cuartiles } & Total \\
\hline 2003 & Q1 & Q2 & Q3 & Q4 & Sin cuartil & \\
\hline 2004 & - & - & - & - & 2 & 2 \\
\hline 2005 & - & - & - & - & 2 & 2 \\
\hline 2006 & - & - & - & - & 1 & 1 \\
\hline 2007 & - & - & - & - & 3 & 3 \\
\hline 2008 & 1 & - & - & - & - & 1 \\
\hline 2009 & 1 & - & 1 & - & - & 2 \\
\hline 2010 & 1 & 1 & & 1 & 2 & 5 \\
\hline 2011 & - & - & 1 & - & 4 & 5 \\
\hline 2012 & 2 & 3 & 2 & 3 & 2 & 10 \\
\hline Total & 6 & 4 & 7 & 4 & 18 & 39 \\
\hline$\%$ & 15 & 10 & 18 & 10 & 46 & 100 \\
\hline
\end{tabular}


Existe un grupo de revistas que no fue posible conocer el cuartil, ya sea por estar inactivas en la base de datos Scopus en el momento de la consulta, o por no constar esta información en el portal SJCR. Dado que una determinada revista puede pertenecer a una o varias categorías de Scopus, el cuartil de publicación (Q1, Q2, Q3 o Q4) obtenido de los sjr de las revistas varía, dependiendo de la categoría seleccionada. En este análisis, siempre se ha tenido en cuenta el cuartil de publicación más alto, entre los que pueda tener una determinada revista, decisión análoga a la recomendada en otros estudios (Ibáñez et al., 2013).

En cuanto a los indicadores relacionados con el impacto real de los trabajos, se determinó que fueron citados en Google Scholar y Scopus un total de 13, lo que representa sólo el 27\%. En la Figura 14 se representa la distribución de los artículos citados por año de publicación, en la que se evidencia que los publicados en el periodo 2006-2008 han sido los más citados.

Figura 14. Distribución del número de artículos citados por año

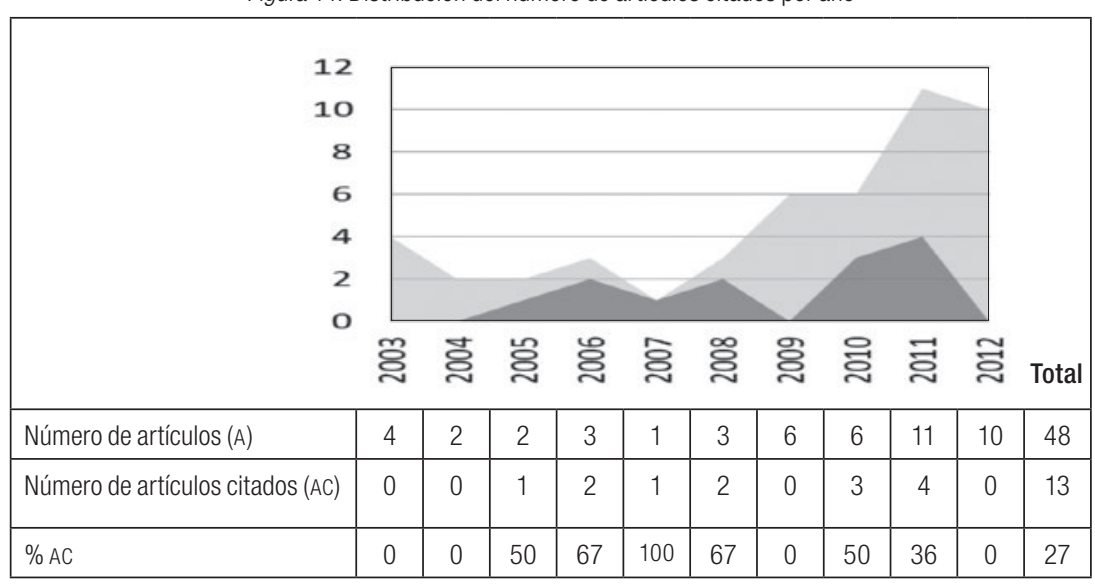

Fuente: elaboración propia, a partir de las fuentes de información descritas en el epígrafe 2.

En la Figura 15, se representa el porcentaje de artículos citados (\% AC), de acuerdo con el tipo de colaboración científica presente en aquéllos, en la cual se evidencia que los artículos publicados en colaboración con autores extranjeros presentaron, de manera general, la mayor cantidad de citas. 
Figura 15. Proporción de artículos citados según el tipo de colaboración

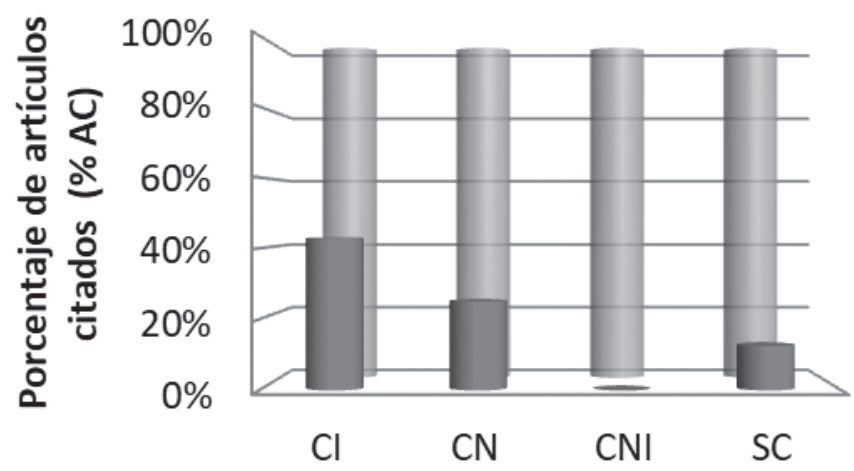

Fuente: elaboración propia a partir de las fuentes de información descritas en el epígrafe 2.

El total de citas recibidas fue 119 (sin eliminar las autocitas), 31 de las cuales son de otras publicaciones de Scopus y 88 de Google Scholar, para un promedio de 0.65 y 1.83 citas por documento, respectivamente.

El cálculo del índice $\mathrm{H}$ dio como resultado que los profesores María Gulnara Baldoquín de la Peña, Rafael Alejandro Espín-Andrade y José Alberto Vilalta-Alonso, los únicos que poseen índice $\mathrm{H}$, según las citas recibidas en Scopus (al igual que los investigadores con mayor número de trabajos en colaboración en el periodo). El resto de los profesores poseen un índice h igual a uno, a partir de las citas recibidas en Google Scholar.

Por último, se realizó un análisis de las referencias bibliográficas de los 31 artículos a texto completo que pudieron recuperarse. Como resultado, se obtuvo que, de un total de 627 referencias, se realizaron 66 citas a otras publicaciones de los profesores de la facultad, de las cuales 60 fueron autocitas; valor que representa un $10 \%$ aproximadamente de autocitas.

\section{Análisis de la contribución de la Facultad a la posición de la institución en el SIR iberoamericano}

En el Cuadro 5 se muestra el análisis realizado, con el objetivo de determinar la contribución de la facultad a la posición de la Cujae en el sIR iberoamericano. 
VISIBILIDAD E IMPACTO DE LA PRODUCCIÓN CIENTÍFICA DE LA FACULTAD DE INGENIERÍA. .

Cuadro 5. Evaluación de los indicadores del SIR iberoamericano para la Facultad de Ingeniería Industrial

\begin{tabular}{|c|c|c|c|c|}
\hline SIR & 2010 & 2011 & 2012 & 2013 \\
\hline Periodos & $2003-2008$ & $2005-2009$ & $2006-2010$ & $2007-2011$ \\
\hline \multicolumn{5}{|c|}{ Producción científica (0) } \\
\hline Cujae & 135 & 150 & 167 & 205 \\
\hline Facultad de Ingeniería Industrial & 11 & 12 & 16 & 23 \\
\hline Porcentaje & 8.15 & 8.00 & 9.58 & 11.22 \\
\hline \multicolumn{5}{|c|}{ Colaboración Internacional (\% IC) } \\
\hline Cujae & 70.37 & 75.3 & 76.7 & 78.54 \\
\hline Facultad de Ingeniería Industrial & 27.27 & 50.00 & 43.75 & 52.17 \\
\hline \multicolumn{5}{|c|}{ Liderazgo científico (\% Lead) } \\
\hline Cujae & & & & 33.17 \\
\hline Facultad de Ingeniería Industrial & & & & 30.43 \\
\hline \multicolumn{5}{|c|}{ Publicaciones de alta calidad (\% Q1) } \\
\hline Cujae & 35.56 & 39.3 & 34.1 & 30.73 \\
\hline Facultad de Ingeniería Industrial & 18.18 & 25.00 & 18.75 & 17.39 \\
\hline IBE & 280 & 334 & 354 & 306 \\
\hline IBE (Facultad) & $489-496$ & $760-777$ & $776-789$ & 448 \\
\hline
\end{tabular}

Fuente: elaboración propia, a partir de las fuentes de información descritas en el epígrafe 2.

Como se aprecia, se compararon los valores relativos a la cantidad de artículos publicados en revistas indizadas en Scopus por la Facultad respecto de la Cujae, en los periodos analizados en las distintas ediciones de dicha base de datos, resultando inferior la producción científica de la facultad en cada reporte. Estos valores la ubican en las posiciones a nivel iberoamericano (IBE) señaladas, si ésta fuera considerada una institución independiente.

En la proporción de trabajos de la facultad en Scopus elaborados conjuntamente con instituciones foráneas, se observó una tendencia al crecimiento, y los valores se mantuvieron por debajo de los logrados por la institución en todos los reportes. En cuanto al porcentaje de la producción en la que la facultad es "el principal contribuidor", éste resultó ser ligeramente inferior al obtenido por la Cujae.

Respecto de las publicaciones de alta calidad, se observa que en todos los periodos el comportamiento fue inferior a los resultados de la institución. Aunque no fue posible el cálculo de la Ratio de Excelencia de la facultad, 
al no contar con información sobre los trabajos más citados en el campo de la Ingeniería Industrial, se determinó que el artículo con mayor número de citas en Scopus (11 citas) lleva por título "Multi-start and path relinking methods to deal with multiobjective knapsack problems", publicado en la revista Annals of Operations Research en 2008, y en el cual participa como coautora María Gulnara Baldoquín de la Peña, en colaboración con autores de la Universidad de La Habana.

Asimismo, no fue posible la determinación del Impacto Normalizado, al ser un indicador comparativo del promedio de citas que recibe una institución con relación al promedio mundial. No obstante, se obtuvo un promedio de citas por artículos publicados en Scopus en el periodo 2007-2011 por la facultad de 1.41.

\section{Discusión}

Resulta importante aclarar que, entre los análisis bibliométricos sobre universidades que fueron estudiados, no se localizó ningún trabajo similar al que se presenta, pues se estudian varias universidades o diferentes bases de datos, o bien se trata de un periodo determinado, una región, un país o una temática concreta, etc. Por tanto, en cada investigación se establecen unos criterios determinados que, por sus características, pueden no ser comparables estrictamente con este estudio, por ello, se tomarán solamente aquellos datos que por algún motivo ofrezcan cierta similitud o diferencia significativa con la Facultad de Ingeniería Industrial.

\section{Indicadores para la dimensión cuantitativa de la producción científica}

La tendencia al crecimiento de la producción científica muestra el esfuerzo sostenido de la Facultad por incrementar su visibilidad en el ámbito internacional y hace pensar en una buena proyección para los años siguientes.

En la revisión de los resultados de diversos estudios bibliométricos (Olmeda et al., 2008; Martí, 2007; Peralta et al., 2011) se observó que en todos, salvo fluctuaciones en años concretos, se produce un incremento anual de documentos publicados, que en ocasiones es más acentuado y en otras más leve, pero siempre ascendente, tanto en ciencia y tecnología, como en ciencias sociales y humanidades (Alonso et al., 2006).

Resulta evidente que los vínculos de colaboración internacional se establecen con investigadores radicados en su mayoría en países de América Latina, con los cuales se tienen establecidas relaciones en el marco de proyectos de investigación conjuntos, así como también vínculos entre los propios investigadores. 
Por otra parte, el predominio del español no está en consonancia con los resultados obtenidos a nivel nacional e internacional, donde predomina la difusión de los resultados de investigación en inglés, con valores por encima del 70\% (Arencibia, 2010; Alonso et al., 2006; Olmeda et al., 2008; Martí, 2007; Peralta et al., 2011).

El análisis de la producción por autores arrojó que el número de autores que produjeron un solo artículo $(59 \%)$ resulta muy similar a los obtenidos en los trabajos de Alonso y colaboradores en la Universidad Politécnica de Valencia y en la Universidad de Extremadura (Alonso et al., 2006). El promedio de artículos por profesor (0.44) y de artículos por doctor (1.26) son ambos valores superiores a la media del sector universitario cubano, obtenida por Arencibia (2010) en el periodo 2006-2007, que fue 0.043 y 0.823 respectivamente.

La contribución de la facultad a la posición de la Cujae en las distintas ediciones del SIR iberoamericano, a partir de los artículos publicados en revistas indizadas en Scopus (que alcanzó un valor del 9\% como promedio), se corresponde con el aporte esperado de acuerdo al número de investigadores de esta facultad.

\section{Indicadores para la colaboración científica}

$\mathrm{Al}$ analizar los indicadores de colaboración científica, para toda la producción de la facultad en revistas de corriente principal en los últimos diez años, se obtuvo un índice de coautoría en el periodo, adecuado a la tendencia descrita en la literatura (Olmeda et al., 2008; Miguel et al., 2006), mientras que en todos los años el número total de artículos publicados en los que al menos un autor pertenece a una institución internacional, superó a los trabajos de autores afiliados a instituciones nacionales (Figura 8); lo cual estaría vinculado con la política de internacionalización de la actividad científica trazada por el MES para la identificación de nuevas fuentes de financiamiento para proyectos, el establecimiento de programas de posgrado conjunto, la creación de grupos de investigación multinacionales y la búsqueda de los canales de comunicación más visibles para la publicación de los resultados de investigación (Arencibia, 2010).

La distribución por centros de educación superior de procedencia de los autores que publicaron, en conjunto con la facultad, es la propia de otras distribuciones de carácter bibliométrico: varias universidades tienen una producción baja y las cinco primeras acumulan el 50\% del total de la producción universitaria. Las tres universidades con las que se colaboró con mayor frecuencia ocupan lugares entre las cien primeras de Iberoamérica, 
correspondientes a España, México y Argentina. Sólo se encuentra presente una universidad no perteneciente a Iberoamérica: Carl von Ossietzky Universitat Oldenburg, de Alemania, con tres documentos en los que participa el mismo autor. De las instituciones cubanas, el protagonismo lo tiene la Universidad de la Habana, en cuanto a volumen y posición en el listado, lo cual coincide con los resultados obtenidos por Arencibia (2010) en el análisis de la colaboración asimétrica entre las universidades de Cuba.

Los resultados del análisis de co-ocurrencia de las palabras clave coinciden con los obtenidos en el examen de la producción por temáticas y permiten concluir que el campo de la Gestión del Conocimiento, en conjunto con la Dirección y la Informática Empresarial, así como la Estadística, son los más frecuentemente abordados en las investigaciones de la Facultad cuyos resultados se divulgan a través de publicaciones en Scopus y Web of Science.

El porcentaje de la producción científica con colaboración internacional en los periodos analizados es inferior en todos los casos al obtenido por la Cujae en las ediciones del SIR iberoamericano, correspondientes a los periodos 2003-2008, 2005-2009, 2006-2010 y 2007-2011; y que se encuentra entre los más altos en relación con el resto de las universidades cubanas. Esto indica que es insuficiente la contribución de la Facultad a los resultados de la institución en este indicador e influye negativamente en la visibilidad e impacto de dichos trabajos.

\section{INDICADORES PARA LA DIMENSIÓN CUALITATIVA DE LA PRODUCCIÓN CIENTÍFICA}

El hecho de que la mayoría de los trabajos se publicaran en revistas extranjeras, se inscribe en la tendencia respecto de los patrones de comunicación de los investigadores de los países de la llamada "ciencia periférica", quienes publican la mayor parte de sus trabajos en revistas de carácter internacional en búsqueda de mayor reconocimiento y visibilidad (Kim y Kim, 2000; Ibáñez et al., 2013).

El porcentaje de artículos en el primer cuartil de la distribución de revistas de Scopus es inferior al obtenido por la Cujae en las distintas ediciones del SIR iberoamericano, y por debajo también del $30.5 \%$ alcanzado por las universidades públicas españolas en el área de las tecnologías informáticas, según el estudio de Ibáñez et al. (2013).

Dichos resultados indican que no se ha priorizado el criterio relativo al cuartil al que pertenecen las revistas en su proceso de selección; lo cual se debería, quizá, al desconocimiento de los autores al respecto y a que este indicador no se encuentra incluido en el sistema de evaluación actual. 
Cabe señalar que, respecto de los indicadores que miden el impacto científico del SIR (el Impacto Normalizado y la Ratio de Excelencia), la Cujae se ha mantenido con niveles bajos en relación con el comportamiento internacional, pero similares a los resultados de las instituciones cubanas.

Aunque no fue posible evaluar la contribución de la facultad respecto de estos indicadores, se concluye que fue bajo el número de citas recibidas por sus trabajos en Scopus, así como que el promedio de citas por documento, tanto a partir de las citas recibidas en Google Scholar como en Scopus, fue pequeño de acuerdo a los resultados obtenidos en diversos estudios de dominio institucional (Arencibia, 2010; Kim y Kim, 2000; Olmeda et al., 2008; Peralta et al., 2011; Ibáñez et al., 2013).

$\mathrm{Al}$ analizar la distribución anual de los valores de este indicador, se obtuvo que en todos los años su comportamiento fue superior en Google Scholar, en comparación con Scopus. Conclusiones similares se han obtenidos en otros estudios y se ha atribuido a causas como las diferencias en la cobertura de documentos (Thornley, 2011; López, 2009) y los conteos de citaciones infladas en Google Scholar (Holden et al., 2010). Llos documentos publicados en colaboración con autores extranjeros presentaron, de manera general, la mayor cantidad de citas (Figura 14); corroborándose los hallazgos de anteriores estudios (Kim y Kim, 2000; Thornley et al., 2011).

Debido a que no fueron detectados estudios en los que se analizaran los valores del índice $\mathrm{H}$ de investigadores en el campo de la Ingeniería Industrial, no se pudo establecer una comparación con los que presentan otros autores. No obstante, según sostiene Hirsch (2005), aun cuando un alto índice $\mathrm{H}$ es un indicador fiable del impacto de un académico, un valor bajo no indica necesariamente lo contrario (Lacasse, 2011). Por último, el valor de las autocitas obtenido se sitúa entre los valores aceptables (10-30\%) estimados para este indicador en dependencia de la disciplina (Wang et al., 2012).

\section{Conclusiones}

El estudio realizado aporta a la Facultad de Ingeniería Industrial información cuantitativa y cualitativa útil para la gestión de sus publicaciones científicas, con vistas al aumento de su visibilidad e impacto a nivel internacional, así como permite contar con mayor información para poder gestionar la producción científica de la Facultad en contribución a los resultados de la institución en el SIR iberoamericano, a la vez que proporciona una herramienta para la toma de decisiones en materia de política científica, la definición de estrategias y la corrección de problemas. 
El análisis de los resultados del estudio bibliométrico permitió concluir que la producción científica de los profesores de la Facultad de Ingeniería Industrial en bases de datos de corriente principal ha presentado una tendencia creciente en los últimos 10 años y su contribución a la posición de la Cujae en el SIR iberoamericano ha sido de un $9 \%$ como promedio.

La mayoría de los artículos analizados fueron publicados en colaboración (nacional o internacional), siendo relativamente bajo el aporte de la Facultad al porcentaje de artículos en colaboración internacional de la Cujae en las distintas ediciones de la clasificación, lo cual influye negativamente en la visibilidad e impacto de dichos trabajos.

El impacto esperado y real de la producción científica estudiada es bajo, ya que los porcentajes de publicaciones de alta calidad de la facultad fueron inferiores a los obtenidos por la institución en el listado o clasificación, así como la cantidad de citas recibidas y el promedio de citas por trabajos publicados en revistas de corriente principal es menor a la tendencia descrita en la literatura, lo cual influye en los indicadores Ratio de Excelencia e Impacto Normalizado.

El procedimiento propuesto puede ser empleado para el estudio de las publicaciones científicas de otras Facultades pertenecientes a universidades cubanas y extranjeras, y se recomienda su aplicación en el resto de las Facultades de la Cujae, con vistas a obtener una imagen más completa del perfil investigativo de la institución.

\section{REFERENCIAS}

Abramo, G., C. Andrea D’Angelo y F. Di Costa. 2009. “Mapping excellence in national research systems: The case of Italy”, Evaluation Review, vol. 33, no. 2: 159188, en <http://erx.sagepub.com/content/33/2/159.abstract>, consultada el 7 de marzo de 2013. DOI: $<10.1177 / 0193841 \times 08322871>$.

Alonso-Arroyo, A., A. Pulgarín e I. Gil-Leiva. 2006. "Análisis bibliométrico de la producción científica de la Universidad Politécnica de Valencia 1973-2001”, Revista Española de Documentación Cientifica, vol. 29, no. 3: 345-363, en <http://redc.revistas.csic.es/index.php/redc/article/view/293/364>, consultada el 29 de septiembre de 2012.

Arencibia, R. 2010. "Visibilidad Internacional de la Ciencia y Educación Superior Cubanas: desafíos del estudio de la producción científica”, Granada-La Habana: Universidad de Granada-Facultad de Comunicación y Documentación Departamento de Biblioteconomía y Documentación/Universidad de La Habana-Facultad de Comunicación Departamento de Ciencias de la Información, tesis doctoral.

Arencibia, R. 2007. "Acimed en Scopus: un nuevo paso hacia la proyección internacional de la investigación cubana sobre bibliotecología y ciencias de la información”, 
Acimed, vol. 16, no. 5, en <http://bvs.sld.cu/revistas/aci/vol16_5_07/aci011107. htm>, consultada el 30 de septiembre de 2012.

Arencibia, R. y J.A. Araújo. 2004. "La producción científica cubana en la bibliografía española de ciencia y tecnología 1995-2001”, Revista Española de Documentación Cientifica, vol. 27, no. 4: 469-481, en <http://redc.revistas.csic.es/index.php/redc/article/view/160/214>, consultada el 29 de septiembre de 2012.

Bernardino, P. y R.C. Marques. 2010. "Academic rankings: an approach to rank portuguese universities”, Ensaio: Avaliação e Políticas Públicas em Educação, vol. 18: 29-48, consultada el 30 de septiembre de 2012, en <http://www.scielo.br/scielo. php?script=sci_arttext $\&$ pid=S0104-40362010000100003 \&nrm $=$ iso $>$.

Campbell, David et al. 2010. "Bibliometrics as a Performance Measurement Tool for Research Evaluation: The Case of Research Funded by the National Cancer Institute of Canada", American Journal of Evaluation, vol. 31, no. 1: 66-83, consultada el 7 de marzo de 2013, en $<10.1177 / 1098214009354774>$.

De Filippo, D., R. Barrere e I. Gómez. 2011. "Características e impacto de la producción científica en colaboración entre Argentina y España”, Revista Iberoamericana de Ciencia, Tecnología y Sociedad, vol. 6, no. 16, en <http://www.scielo.org.ar/ scielo.php?script $=$ sci_pdf\&pid $=$ S1850-00132011000100009\&lng $=e s \& n r m=i-$ so\&tlng=es $>$, consultada el 30 de septiembre de 2012.

Dirección de Política Científica y Tecnológica, Citma. 2001. Documentos rectores de la Ciencia y la Innovación Tecnológica. La Habana: Academia.

González Guitián, María Virginia y Maricela Molina Piñeiro. 2008. "La evaluación de la ciencia y la tecnología: revisión de sus indicadores", Acimed, vol. 18, no. 6.

Holden, G., G. Rosenberg, K. Barker y J. Lioi. 2010. "Research on Social Work Practice: A Bibliometric Evaluation of the First Decade", Research on Social Work Practice, vol. 20, no. 1: 11-20, en <10.1177/1049731509332877>; <http://rsw.sagepub.com/content/20/1/11.abstract $>$, consultada el 7 de marzo de 2013.

Ibáñez, A., C. Bielza y P. Larrañaga. 2013. "Análisis de la actividad científica de las universidades públicas españolas en el área de las tecnologías informáticas", Revista Española de Documentación Cientifica, vol. 36, no. 1: 1-17, en <http://redc. revistas.csic.es/index.php/redc/article/view/772/860>, consultada el 2013-0321], ISSN 0210-0614. DOI: <http://dx.DOI.org/10.3989/redc.2013.1.912>.

Ingeniería Industrial, Instituto Superior Politécnico "José Antonio Echeverría, Cujae". 2013. "Política editorial", en <http://rii.cujae.edu.cu/index.php/revistaind/ about/editorialPolicies\#focusAndScope>, consultada el 26 de agosto de 2013.

Kim, M.J. y B.J. Kim. 2000. "A bibliometric analysis of publications by the Chemistry Department, Seoul National University, Korea, 1992-1998”, Journal of Information Science, vol. 26, no. 2: 111-119, en <http://jis.sagepub.com/content/26/2/111. abstract>, consultada el 7 de marzo de 2013. DOI: $<10.1177 / 01655515000260$ 0204>.

Kumar, S. y Shehbaz Husain N. 2010. "Research output in the field of natural sciences: A bibliometric case study of Jamia Millia Islamia University, New Delhi”, IFLA Journal, vol. 36, no. 4: 317-324, en <http://ifl.sagepub.com/content/36/4/317.abstract>, consultada el 7 de marzo de 2013. DOI: <10.1177/ 0340035210388242>.

Lacasse, J.R.; D. R. Hodge y K.F. Bean. 2011. "Evaluating the productivity of social work scholars using the h-Index", Research on Social Work Practice, vol. 21, no. 5: 
599-607, en <http://rsw.sagepub.com/content/21/5/599.abstract>, consultada el 7 de marzo de 2013. DOI: <10.1177/1049731511405069>.

López-Illescas, C., F. de Moya Anegón y H.F. Moed. 2009. “Comparing bibliometric country-by-country rankings derived from the Web of Science and Scopus: the effect of poorly cited journals in oncology", Journal of Information Science, vol. 35, no. 2: 244-256, en <http://jis.sagepub.com/content/35/2/244.abstract>, consultada el 7 de marzo de 2013. DOI: $<10.1177 / 0165551508098603>$.

Martí, Y. 2007. "Análisis de la producción científica de la Universidad de La Habana: una aproximación desde los patrones de comunicación multi e interdisciplinar de sus profesores e investigadores en la Web de la Ciencia (1988-2006)”. La Habana: Universidad de La Habana-Universidad de Granada, Diploma de Estudios Avanzados. Programa Doctoral en Documentación e Información Científica.

Miguel, S., F. de Moya-Anegón y V. Herrero-Solana. 2012. "Aproximación metodológica para la identificación del perfil y patrones de colaboración de dominios científicos universitarios", Revista Española de Documentación Científica, vol. 29, no. 1, consultada el 29 de septiembre de 2012, en <http://redc.revistas.csic.es/index. $\mathrm{php} / \mathrm{redc} /$ article/view/286/343>.

Miguel, S., F. de Moya-Anegón y V. Herrero-Solana. 2006. "Aproximación metodológica para la identificación del perfil y patrones de colaboración de dominios científicos universitarios", Revista Española de Documentación Cientifica, vol. 29, no. 1: 36-55, en <http://redc.revistas.csic.es/index.php/redc/article/view/286/ 343>, consultada el 29 de septiembre de 2012.

Moya-Anegón, F., Z. Chinchilla-Rodríguez, E. Corera-Álvarez, B. Vargas-Quesada, F. Muñoz-Fernández y V. Herrero-Solana. 2005. "Análisis de dominio institucional: la producción científica de la Universidad de Granada (SCI 1991-99)”, Revista Española de Documentación Cientifica, vol. 28, no. 2: 170-195, en <http://redc.revistas.csic.es/index.php/redc/article/view/167/221>, consultada el 29 de septiembre de 2012.

Olmeda-Gómez, C., M.A. Ovalle-Perandones, A. Perianes-Rodríguez y F. de Moya-Anegón. 2008. "Impacto internacional de la investigación y la colaboración científica de las Universidades de Cataluña. 2000-2004”, Revista Española de Documentación Cientifica, vol. 31, no. 4:591-611. DOI: <10.3989/redc.2008.4.659>.

Peralta, M. J., F.M. Solís y L.M. Peralta. 2011. "Visibilidad e impacto de la producción científica de la Universidad Central 'Marta Abreu' de Las Villas durante el periodo 2000-2008”, Acimed, vol. 22, no. 1: 60-78, en <http://scielo.sld.cu/scielo. php? script=sci_arttext\&pid=S1024-94352011000100006\&nrm=iso $>$, consultada el 30 de septiembre de 2012.

Phelan, T.J. 2000. "Bibliometrics and the evaluation of Australian sociology", Journal of Sociology, vol. 36, no. 3: 345-363, en <http://jos.sagepub.com/content/36/ 3/345.abstract $>$, consultada el 7 de marzo de 2013. DOI: $<10.1177 / 144078$ $330003600305>$.

Sánchez Tarragó, N. 2007. "La comunicación de la ciencia en los países en vías de desarrollo y el Movimiento Open Acces”, Biblios, vol. 8, no. 27, en <http://dialnet. unirioja.es/descarga/articulo/2281804.pdf $>$, consultada el 8 de marzo.

Sanz, E. et al. 2005. "Análisis de la actividad científica de la Facultad de Humanidades de la Universidad de Mar del Plata, durante el periodo 1998-2001”, Revista Española de Documentación Científica, vol. 28, no. 2: 196-205, en <http://redc. 
revistas.csic.es/index.php/redc/article/view/168/222>, consultada el 29 de septiembre de 2012.

Schäffer, U. y C. Binder. 2008. “'Controlling’ as an academic discipline: the development of management accounting and management control research in German-speaking countries between 1970 and 2003", Accounting History, vol. 13, no. 1: 33-74, en <http://ach.sagepub.com/content/13/1/33.abstract>, consultada el 7 de marzo de 2013. DOI: <10.1177/1032373207083926>.

Scimago Research Group (SRG). 2013. "Scimago Institutions Ranking”, en <http:// www.scimagoir.com/index.php>, consultada el 11 de septiembre de 2013.

Thornley, C.V., S.J. McLoughlin, A.C. Johnson y A.F Smeaton. 2011. "A bibliometric study of Video Retrieval Evaluation Benchmarking (TRECVid): A methodological analysis", Journal of Information Science, vol. 37, no. 6: 577-593, en $<$ http://jis.sagepub.com/content/37/6/577.abstract $>$, consultada el 7 de marzo de 2013. DOI: $<10.1177 / 0165551511420032>$.

Torres-Salinas, D. y E. Jiménez-Contreras. 2012. "Hacia las unidades de bibliometría en las universidades: modelo y funciones”, Revista Española de Documentación Cientifica, vol. 35, no. 3 (julio-septiembre): 469-480. DOI: <10.3989/redc.2012.3. 959>.

Wang, G.G., J.W. Gilley y J.Y. Sun. 2012. “The ‘Science of HRD Research’: Reshaping HRD Research Through Scientometrics”, Human Resource Development Review, vol. 11, no. 4: 500-520, en <10.1177/1534484312452265>; <http://hrd.sagepub. com/content/11/4/500.abstract $>$, consultada el 7 de marzo de 2013.

\section{Para citar este texto:}

Fleitas Triana, María Sonia; Hernández Oquendo, Claudia; Guerra Castillo, Sandra. 2017. "Visibilidad e impacto de la producción científica de la Facultad de Ingeniería Industrial de la Cujae de Cuba 2003-2012”. Investigación Bibliotecológica: Archivonomía, Bibliotecología e Información (Número Especial de Bibliometría), 2017: 149-185.

http://dx.doi.org/10.22201/iibi.24488321xe.2017.nesp1.57889 